Annals of Pure and Applied Mathematics

Vol. 12, No. 2, 2016, 169-176

ISSN: 2279-087X (P), 2279-0888(online)

Published on 17 November 2016

www.researchmathsci.org

DOI: http://dx.doi.org/10.22457/apam.v12n2a8

Annals of

Pure and Applied

Mathematics

\title{
Multiplicative Connectivity Indices of Certain Nanotubes
}

\author{
V.R.Kulli \\ Department of Mathematics \\ Gulbarga University, Gulbarga 585106, India \\ e-mail: vrkulli@gmail.com
}

Received 5 November 2016; accepted 16 November 2016

Abstract. In this paper, we introduce some multiplicative connectivity indices of a graph. A topological index is a numeric quantity from the structural graph of a molecule. In this paper, we compute first multiplicative Zagreb index, multiplicative hyper-Zagreb, general multiplicative Zagreb, multiplicative sum connectivity, multiplicative product connectivity, multiplicative $A B C$, general multiplicative $G A$ indices for certain important chemical structures like nanotubes covered by $\mathrm{C}_{5}$ and $\mathrm{C}_{7}$

Keywords: molecular graph, multiplicative indices, nanotubes

AMS Mathematics Subject Classification (2010): 05C05, 05C07, 05C35

\section{Introduction}

In this paper, we consider only finite connected, undirected without loops and multiple edges. Let $G$ be a connected graph with vertex set $V(G)$ and edge set $E(G)$. The degree $d_{G}(v)$ of a vertex $v$ is the number of vertices adjacent to $v$. The edge connecting the vertices $u$ and $v$ will be denoted by $u v$. For other undefined notations and terminology, the readers are referred to [1].

A molecular graph is a simple graph such that its vertices correspond to the atoms and the edges to the bonds. Chemical graph theory is a branch of mathematical chemistry which has an important effect on the development of the chemical sciences. A single number that can be used to characterize some property of the graph of a molecular is called a topological index for that graph. In organic chemistry, topological indices have been found to be useful in chemical documentation, isomer discrimination, structure property relationships, structure activity relationships and pharmaceutical drug design. There has been considerable interest in the general problem of determining topological indices.

The first and second multiplicative Zagreb indices of a graph $G$ are defined as

$$
I I_{1}(G)=\prod_{u \in V(G)} d_{G}(u)^{2}, \quad I I_{2}(G)=\prod_{u v E(G)} d_{G}(u) d_{G}(v) .
$$

These indices were introduced by Todeshine et al. in [2] and were studied, for example, in $[3,4,5,6,7,8,9,10,11]$. 

index as

In [12], Eliasi et al. proposed a new multiplicative version of the first Zagreb

$$
I I_{1}^{*}(G)=\prod_{u v \in E(G)}\left[d_{G}(u)+d_{G}(v)\right] .
$$

The first and second multiplicative hyper-Zagreb indices of a graph $G$ are defined as

$$
H I_{1}(G)=\prod_{u v \in E(G)}\left[d_{G}(u)+d_{G}(v)\right]^{2}, H I_{2}(G)=\prod_{u v \in E(G)}\left[d_{G}(u) d_{G}(v)\right]^{2} .
$$

These indices were introduced by Kulli in [13]. defined as

The general first and second multiplicative Zagreb indices of a graph $G$ are

$$
M Z_{1}^{a}(G)=\prod_{u v \in E(G)}\left[d_{G}(u)+d_{G}(v)\right]^{a}, M Z_{2}^{a}(G)=\prod_{u v \in E(G)}\left[d_{G}(u) d_{G}(v)\right]^{a} .
$$

These topological indices were introduced by Kulli et al. in [14].

One of the best known and widely used topological index is the product connectivity index or Randić index, introduced by Randić in [15]. The product connectivity index of a graph $G$ is defined as

$$
\chi(G)=\sum_{u v \in E(G)} \frac{1}{\sqrt{d_{G}(u) d_{G}(v)}} .
$$

Motivated by the definition of the product connectivity index and its wide applications, we introduce the multiplicative product connectivity index, multiplicative sum connectivity index, multiplicative atom bond connectivity index, multiplicative geometric-arithmetic index and also general multiplicative geometric-arithmetic index of a graph as follows:

The multiplicative sum connectivity index of a graph $G$ is defined as

$$
X I I(G)=\prod_{u v \in E(G)} \frac{1}{\sqrt{d_{G}(u)+d_{G}(v)}} .
$$

The multiplicative product connectivity index of a graph $G$ is defined as

$$
\chi I I(G)=\prod_{u v \in E(G)} \frac{1}{\sqrt{d_{G}(u) d_{G}(v)}} .
$$

The multiplicative atom bond connectivity index of a graph $G$ is defined as

$$
A B C I I(G)=\prod_{u v \in(G)} \sqrt{\frac{d_{G}(u)+d_{G}(v)-2}{d_{G}(u) d_{G}(v)}} .
$$

The multiplicative geometric-arithmetic index of a graph $G$ is defined as

$$
G A I I(G)=\prod_{u v \in E(G)} \frac{2 \sqrt{d_{G}(u) d_{G}(v)}}{d_{G}(u)+d_{G}(v)} .
$$
follows:

The general multiplicative geometric-arithmetic index of a graph $G$ is defined as 
Multiplicative Connectivity Indices of Certain Nanotubes

$$
G A^{a} I I(G)=\prod_{u v E(G)}\left(\frac{2 \sqrt{d_{G}(u) d_{G}(v)}}{d_{G}(u)+d_{G}(v)}\right)^{a} .
$$

Nanotubes are basic and primal structures of other more complicated chemical molecular structures. These molecular structures have been widely applied in chemical engineering, medical science and pharmaceutical fields. Therefore we compute multiplicative connectivity indices of nanotubes covered by $C_{5}$ and $C_{7}$ with industries and academic interest.

\section{Results for nanotubes covered by $\boldsymbol{C}_{5}$ and $\boldsymbol{C}_{7}$}

We compute index for certain special classes of nanotubes, viz, $V C_{5} C_{7}[p, q]$ and $H C_{5} C_{7}[p, q]$ nanotubes. These nanotubes are trivalent decoration constructed by $C_{5}$ and $C_{7}$ in turn and they can cover either a cylinder or a torus. The parameter $p$ is the number of pentagons in the first row of $V C_{5} C_{7}[p, q]$ and $H C_{5} C_{7}[p, q]$. The vertices and edges in first four rows are repeated alternately, we denote the number of this repetition by $q$.

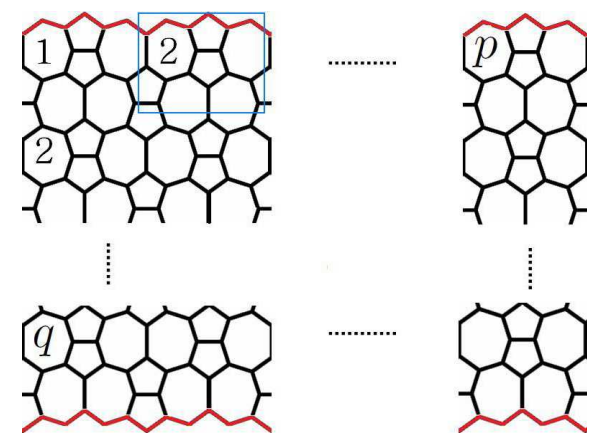

Figure 1: $V C_{5} C_{7}[p, q]$ nanotube
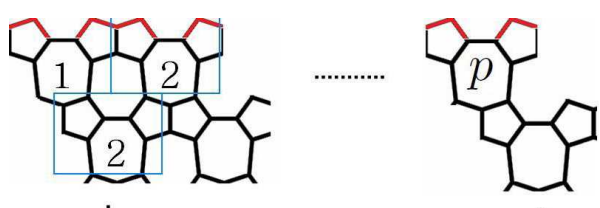

:
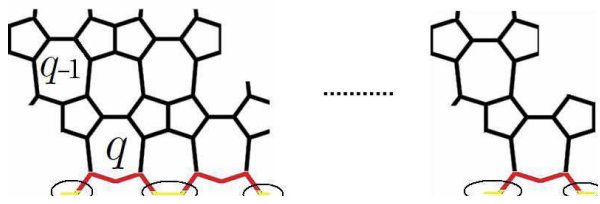

Figure 2: $H C_{5} C_{7}[p, q]$ nanotube 
Theorem 2.1. Let $G=V C_{5} C_{7}[p, q]$ and $G_{1}=H C_{5} C_{7}[p, q]$ be two classes of nanotubes. Then

$$
\text { 1) } \quad I I_{1}\left(V C_{5} C_{7}[p, q]\right)=2^{12 p} 3^{32 p q} .
$$

Proof: 1) Let $G=V C_{5} C_{7}[p, q]$ be the nanotubes. By algebraic method, we get $|V(G)|=16 p q+6 p \mid$ and $E(G) \mid=24 p q+6 p$. We have two partitions of the vertex set $V(G)$ as follows:

$$
\begin{array}{llrl}
V_{2} & =\left\{v \in V(G): d_{G}(v)=2\right\}, & & \left|V_{2}\right|=6 p . \\
V_{3}=\left\{v \in V(G): d_{G}(v)=3\right\}, & & \left|V_{3}\right|=16 p q .
\end{array}
$$

Now

$$
\begin{aligned}
I I_{1}(G) & =\prod_{u \in V(G)} d_{G}(u)^{2}=\prod_{u \in V_{2}} 2^{2} \times \prod_{u \in V_{3}} 3^{2}=\left(2^{2}\right)^{6 p} \times\left(3^{2}\right)^{16 p q} \\
& =2^{12 p} 3^{32 p q} .
\end{aligned}
$$

2) Let $G_{1}=H C_{5} C_{7}[p, q]$ be the nanotubes. By algebraic method, we get $\left|V\left(G_{1}\right)\right|=8 p q+5 p$ and $\left|E\left(G_{1}\right)\right|=12 p q+5 p$. We have two partitions of the vertex set $V\left(G_{1}\right)$ as follows:

$$
\begin{array}{llrl}
V_{2} & =\left\{v \in V\left(G_{1}\right): d_{G}(v)=2\right\}, & & \left|V_{2}\right|=5 p . \\
V_{3} & =\left\{v \in V\left(G_{1}\right): d_{G}(v)=3\right\}, & & \left|V_{3}\right|=8 p q .
\end{array}
$$

Now

$$
\begin{aligned}
I I_{1}\left(G_{1}\right) & =\prod_{\substack{u \in V\left(G_{1}\right) \\
G_{G_{1}}}}(u)^{2}=\prod_{u \in V_{2}} 2^{2} \times \prod_{u \in V_{3}} 3^{2}=\left(2^{2}\right)^{5 p} \times\left(3^{2}\right)^{12 p q} \\
& =2^{10 p} 3^{24 p q} .
\end{aligned}
$$

We now determine the first and second multiplicative hyper-Zagreb indices of nanotubes.

Theorem 2.2. Let $G=V C_{5} C_{7}[p, q]$ and $G_{1}=H C_{5} C_{7}[p, q]$ be two classes of nanotubes. Then
1) $\quad H I_{1}\left(V C_{5} C_{7}[p, q]\right)=5^{24 P} 6^{48 p q-12 p}$.
2) $\quad \mathrm{HII}_{2}\left(V C_{5} C_{7}[p, q]\right)=6^{24 P} 9^{48 p q-12 p}$
3) $\quad H I_{1}\left(H C_{5} C_{7}[p, q]\right)=4^{2 p} 5^{16 p} 6^{24 p q-8 p}$.
4) $\quad \mathrm{HII}_{2}\left(H \mathrm{HC}_{5} \mathrm{C}_{7}[p, q]\right)=4^{2 p} 6^{16 p} 9^{24 p q-8 p}$.

Proof: Let $G=V C_{5} C_{7}[p, q]$ be the nanotubes. By algebraic method, we get $|V(G)|=16 p q+6 p$ and $|E(G)|=24 p q+6 p$. We have two partitions of the edge set $E(G)$ as given in Table 1.

\begin{tabular}{ccl}
\hline$d_{G}(u), d_{G}(u) \backslash u v \in E(G)$ & $E_{5}=(2,3)$ & $E_{6}=(3,3)$ \\
\hline Number of edges & $12 p$ & $24 p q-6 p$ \\
\hline
\end{tabular}

Table 1: Computing the number of edges for $V C_{5} C_{7}[p, q]$ nanotube.

1)

$$
\begin{aligned}
H I I_{1}(G) & =\prod_{\substack{u \in E(G)\\
}}\left[d_{G}(u)+d_{G}(v)\right]^{2}=\prod_{u v \in E_{5}}(2+3)^{2} \times \prod_{u v \in E_{6}}(3+3)^{2} \\
& =5^{24 p} 6^{48 p q-12 p} .
\end{aligned}
$$


Multiplicative Connectivity Indices of Certain Nanotubes

2)

$$
\begin{aligned}
H I_{2}(G) & =\prod_{u v \in E(G)}\left[d_{G}(u) d_{G}(v)\right]^{2}=\prod_{u v \in E_{5}}(2 \times 3)^{2} \times \prod_{u v \in E_{6}}(3 \times 3)^{2} \\
& =6^{24 p} 9^{48 p q-12 p} .
\end{aligned}
$$

Let $G_{1}=\mathrm{HC}_{5} \mathrm{C}_{7}[p, q]$ be the nanotubes. By algebraic method, we get $\left|V\left(G_{1}\right)\right|=8 p q+5 p$ and $\left|E\left(G_{1}\right)\right|=12 p q+5 p$. We have three partitions of the edge set $E\left(G_{1}\right)$ as given in Table 2.

\begin{tabular}{cccc}
\hline$d_{G}(u), d_{G}(u) \backslash u v \in E(G)$ & $E_{4}=(2,2)$ & $E_{5}=(2,3)$ & $E_{6}=(3,3)$ \\
\hline Number of edges & $P$ & $8 p$ & $12 p q-4 p$ \\
\hline
\end{tabular}

Table 2: Computing the number of edges for $\mathrm{HC}_{5} \mathrm{C}_{7}[p, q]$ nanotube.

$$
\begin{aligned}
& \text { 3) } H I I_{1}\left(G_{1}\right)=\prod_{u v \in\left(G_{1}\right)}\left[d_{G_{1}}(u)+d_{G_{1}}(v)\right]^{2}=\prod_{u v \in E_{4}}(2+2)^{2} \times \prod_{u v \in E_{5}}(2+3)^{2} \times \prod_{u v \in E_{6}}(3+3)^{2} \\
& =4^{2 p} 5^{16 p} 6^{24 p q-8 p} \text {. } \\
& \text { 4) } H I I_{2}\left(G_{1}\right)=\prod_{u v \in E\left(G_{1}\right)}\left[d_{G_{1}}(u) d_{G_{1}}(v)\right]^{2}=\prod_{u v \in E_{4}}(2 \times 2)^{2} \times \prod_{u v \in E_{5}}(2 \times 3)^{2} \times \prod_{u v \in E_{6}}(3 \times 3)^{2} \\
& =4^{2 p} 6^{16 p} 9^{24 p q-8 p} \text {. }
\end{aligned}
$$

Theorem 2.3. Let $G=V C_{5} C_{7}[p, q]$ be a class of nanotubes. Then

1) $\quad X I I\left(V C_{5} C_{7}[p, q]\right)=\left(\frac{1}{5}\right)^{6 p}\left(\frac{1}{6}\right)^{12 p q-3 p}$.

2) $\quad \chi I I\left(V C_{5} C_{7}[p, q]\right)=\left(\frac{1}{\sqrt{6}}\right)^{12 p}\left(\frac{1}{3}\right)^{24 p q-6 p}$.

3) $\quad M Z_{1}^{a}\left(V C_{5} C_{7}[p, q]\right)=5^{12 p a} 6^{(24 p q-6 p) a}$.

4) $\quad M Z_{2}^{a}\left(V C_{5} C_{7}[p, q]\right)=6^{12 p a} 9^{(24 p q-6 p) a}$.

Proof: By using Table 1, we get

1) $\quad X I I(G)=\prod_{u \in E(G)} \frac{1}{\sqrt{d_{G}(u)+d_{G}(v)}}=\prod_{u v \in E_{5}} \frac{1}{\sqrt{2+3}} \times \prod_{u v \in E_{6}} \frac{1}{\sqrt{3+3}}$

$$
=\left(\frac{1}{\sqrt{5}}\right)^{12 p} \times\left(\frac{1}{\sqrt{6}}\right)^{24 p q-6 p}=\left(\frac{1}{5}\right)^{6 p} \times\left(\frac{1}{6}\right)^{12 p q-3 p} .
$$

2) $\quad \chi I I(G)=\prod_{u v E(G)} \frac{1}{\sqrt{d_{G}(u) d_{G}(v)}}=\prod_{u v \in E_{5}} \frac{1}{\sqrt{2 \times 3}} \times \prod_{u v \in E_{6}} \frac{1}{\sqrt{3 \times 3}}$

$$
=\left(\frac{1}{\sqrt{6}}\right)^{12 p} \times\left(\frac{1}{3}\right)^{24 p q-6 p} \text {. }
$$


V.R.Kulli

3) $\quad M Z_{1}^{a}(G)=\prod_{u v \in E(G)}\left[d_{G}(u)+d_{G}(v)\right]^{a}=5^{12 p a} \times 6^{(24 p q-6 p) a}$.

4) $\quad M Z_{2}^{a}(G)=\prod_{u v E(G)}\left[d_{G}(u) d_{G}(v)\right]^{a}=6^{12 p a} \times 9^{(24 p q-6 p) a}$.

Theorem 2.4. Let $G_{1}=H C_{5} C_{7}[p, q]$ be a class of nanotubes. Then

1) $\quad X I I\left(H C_{5} C_{7}[p, q]\right)=\left(\frac{1}{\sqrt{4}}\right)^{p}\left(\frac{1}{\sqrt{5}}\right)^{8 p}\left(\frac{1}{\sqrt{6}}\right)^{12 p q-4 p}$.

2) $\quad \chi I I\left(H C_{5} C_{7}[p, q]\right)=\left(\frac{1}{2}\right)^{p}\left(\frac{1}{\sqrt{6}}\right)^{8 p}\left(\frac{1}{3}\right)^{12 p q-4 p}$.

3) $\quad M Z_{1}^{a}\left(H C_{5} C_{7}[p, q]\right)=4^{p a} 5^{8 p a} 6^{(12 p q-4 p) a}$.

4) $\quad M Z_{2}^{a}\left(H C_{5} C_{7}[p, q]\right)=4^{p a} 6^{8 p a} 9^{(12 p q-4 p) a}$.

Proof: By using Table 2, we get

1) $\quad X I I\left(G_{1}\right)=\prod_{u \in E\left(G_{1}\right)} \frac{1}{\sqrt{d_{G_{1}}(u)+d_{G_{1}}(v)}}=\prod_{u v \in E_{4}} \frac{1}{\sqrt{2+2}} \times \prod_{u v \in E_{5}} \frac{1}{\sqrt{2+3}} \times \prod_{u v \in E_{6}} \frac{1}{\sqrt{3+3}}$

$$
=\left(\frac{1}{\sqrt{4}}\right)^{p} \times\left(\frac{1}{\sqrt{5}}\right)^{8 p} \times\left(\frac{1}{\sqrt{6}}\right)^{12 p q-4 p} \text {. }
$$

2) $\quad \chi I I\left(G_{1}\right)=\prod_{u v \in E\left(G_{1}\right)} \frac{1}{\sqrt{d_{G_{1}}(u) d_{G_{1}}(v)}}=\prod_{u v \in E_{4}} \frac{1}{\sqrt{2 \times 2}} \times \prod_{u v \in E_{5}} \frac{1}{\sqrt{2 \times 3}} \times \prod_{u v \in E_{6}} \frac{1}{\sqrt{3 \times 3}}$

$$
=\left(\frac{1}{2}\right)^{p} \times\left(\frac{1}{\sqrt{6}}\right)^{8 p} \times\left(\frac{1}{3}\right)^{12 p q-4 p} \text {. }
$$

3) $\quad M Z_{1}^{a}\left(G_{1}\right)=\prod_{u v E\left(G_{1}\right)}\left[d_{G_{1}}(u)+d_{G_{1}}(v)\right]^{a}=4^{p a} 5^{8 p a} 6^{(12 p q-4 p) a}$.

4) $\quad M Z_{2}^{a}\left(G_{1}\right)=\prod_{u v E\left(G_{1}\right)}\left[d_{G_{1}}(u) d_{G_{1}}(v)\right]^{a}=4^{p a} 5^{8 p a} 6^{(12 p q-4 p) a}$.

Theorem 2.5. Let $G=V C_{5} C_{7}[p, q]$ and $G_{1}=H C_{5} C_{7}[p, q]$ be two classes of nanotubes. Then

1) $\quad A B C I I\left(V C_{5} C_{7}[p, q]\right)=2^{24 p q-12 p} 3^{6 p-24 p q}$

2) $\quad A B C I I\left(H C_{5} C_{7}[p, q]\right)=2^{12 p q-\frac{17}{2} p} 3^{4 p-12 p q}$.

Proof:1) By using Table 1, we get

$$
A B C I I(G)=\prod_{u v \in E(G)} \sqrt{\frac{d_{G}(u)+d_{G}(v)-2}{d_{G}(u) d_{G}(v)}}=\prod_{u v \in E_{5}} \sqrt{\frac{2+3-2}{2 \times 3}} \times \prod_{u v \in E_{5}} \sqrt{\frac{3+3-2}{3 \times 3}}
$$


Multiplicative Connectivity Indices of Certain Nanotubes

$$
=\left(\frac{1}{\sqrt{2}}\right)^{12 p}\left(\frac{2}{3}\right)^{24 p q-6 p}=2^{24 p q-12 p} 3^{6 p-24 p q} .
$$

2) By using table 2, we get,

$$
\begin{aligned}
A B C I I\left(G_{1}\right) & =\prod_{u \in \in E\left(G_{1}\right)} \sqrt{\frac{d_{G_{1}}(u)+d_{G_{1}}(v)-2}{d_{G_{1}}(u) d_{G_{1}}(v)}}=\prod_{u \in E_{4}} \sqrt{\frac{2+2-2}{2 \times 2}} \times \prod_{u \in \in E_{5}} \sqrt{\frac{2+3-2}{2 \times 3}} \times \prod_{u \in E_{6}} \sqrt{\frac{3+3-2}{3 \times 3}} \\
& =\left(\frac{1}{\sqrt{2}}\right)^{p}\left(\frac{1}{\sqrt{2}}\right)^{8 p}\left(\frac{2}{3}\right)^{12 p q-6 p}=2^{12 p q-\frac{17}{2} p} 3^{4 p-12 p q} .
\end{aligned}
$$

Theorem 2.6. Let $G=V C_{5} C_{7}[p, q]$ and $G_{1}=H C_{5} C_{7}[p, q]$ be two classes of nanotubes. Then
1) $\quad \operatorname{GAII}\left(V C_{5} C_{7}[p, q]\right)=\left(\frac{2 \sqrt{6}}{5}\right)^{12 p}$.
2) $\quad G A^{a} I I\left(V C_{5} C_{7}[p, q]\right)=\left(\frac{2 \sqrt{6}}{5}\right)^{12 p a}$.
3) $\quad \operatorname{GAII}\left(H \mathrm{HC}_{5} C_{7}[p, q]\right)=\left(\frac{2 \sqrt{6}}{5}\right)^{8 p}$.
4) $\quad G A^{a} I I\left(H C_{5} C_{7}[p, q]\right)=\left(\frac{2 \sqrt{6}}{5}\right)^{8 p a}$.

Proof: 1) By using Table 1, we get

$$
\begin{aligned}
\operatorname{GAII}(G) & =\prod_{u v \in E(G)} \frac{2 \sqrt{d_{G}(u) d_{G}(v)}}{d_{G}(u)+d_{G}(v)}=\prod_{u v \in E_{5}} \frac{2 \sqrt{2 \times 3}}{2+3} \times \prod_{u v \in E_{6}} \frac{2 \sqrt{3 \times 3}}{3+3} \\
& =\left(\frac{2 \sqrt{6}}{5}\right)^{12 p} \times(1)^{24 p q-6 p}=\left(\frac{2 \sqrt{6}}{5}\right)^{12 p} . \\
\text { 2) } \quad G A^{a} I I(G) & =\prod_{u v E(G)}\left(\frac{2 \sqrt{d_{G}(u) d_{G}(v)}}{d_{G}(u)+d_{G}(v)}\right)^{a}=\left(\frac{2 \sqrt{6}}{5}\right)^{12 p a} .
\end{aligned}
$$

3) By using Table 2, we get

$$
\begin{aligned}
\operatorname{GAII}\left(G_{1}\right) & =\prod_{u v \in E\left(G_{1}\right)} \frac{2 \sqrt{d_{G_{1}}(u) d_{G_{1}}(v)}}{d_{G_{1}}(u)+d_{G_{1}}(v)}=\prod_{u v \in E_{4}} \frac{2 \sqrt{2 \times 2}}{2+2} \times \prod_{u v \in E_{5}} \frac{2 \sqrt{2 \times 3}}{2+3} \times \prod_{u v \in E_{6}} \frac{2 \sqrt{3 \times 3}}{3+3} \\
= & (1)^{p} \times\left(\frac{2 \sqrt{6}}{5}\right)^{8 p} \times(1)^{12 p q-4 p}=\left(\frac{2 \sqrt{6}}{5}\right)^{8 p} .
\end{aligned}
$$


4) $\quad G A^{a} I I\left(G_{1}\right)=\prod_{u v E E\left(G_{1}\right)}\left(\frac{2 \sqrt{d_{G_{1}}(u) d_{G_{1}}(v)}}{d_{G_{1}}(u)+d_{G_{1}}(v)}\right)^{a}=\left(\frac{2 \sqrt{6}}{5}\right)^{8 p a}$.

\section{REFERENCES}

1. V.R.Kulli, College Graph Theory, Vishwa International Publications, Gulbarga, India 2012.

2. R.Todeshine and V.Consonni, New local vertex invariants and descriptors based on functions of vertex degrees, MATCH Commun. Math. Comput. Chem., 64 (2010) 359-372.

3. K.C.Das, A.Yurttas, M.Togan, A.S.Cevik and N.Cangul, The multiplicative Zagreb indices of graph operations, Journal of Inequalities and Applications, 90 (2013) 1-14.

4. F.Falahati Nezhad, A.Iranmanesh, A.Tehranian and M.Azari, Comparing the second multiplicative Zagreb coindex with some graph invariants, Trans. Comb. 3(4) (2014) 31-41.

5. I.Gutman, Multiplicative Zagreb indices of trees, Bull. Soc. Math. Banja Luka, 18(2011) 17-23.

6. V.R.Kulli, First multiplicative $K$ Banhatti index and coindex of graphs, Annals of Pure and Applied Mathematics, 11(2) (2016) 79-82.

7. V.R.Kulli, Second multiplicative $K$ Banhatti index and coindex of graphs, Journal of Computer and Mathematical Sciences, 7(5) (2016) 254-258.

8. V.R.Kulli, On $K$ hyper-Banhatti indices and coindices of graphs, International Journal of Mathematical Archive, 7(6) (2016) 60-65.

9. V.R.Kulli, On multiplicative $K$ Banhatti and multiplicative $K$ hyper-Banhatti indices of $V$-Phenylenic nanotubes and nanotorus, Annals of Pure and Applied Mathematics, 11(2) (2016) 145-150.

10. H.Wang and H.Bao, A note on multiplicative sum Zagreb index, South Asian J. Math. 2(6) (2012) 578-583.

11. S.Wang and B.Wei, Multiplicative Zagreb indices of $k$-trees, Discrete Applied Math. 180 (2015) 168-175.

12. M.Eliasi, A.Iranmanesh and I.Gutman, Multiplicative versions of first Zagreb index, MATCH Commun. Math. Comput. Chem. 68(2012) 217-230.

13. V.R.Kulli, Multiplicative hyper-Zagreb indices and coindices of graphs, Intern. Journal of Pure Algebra, 6(7) (2016) 342-347.

14. V.R.Kulli, B.Stone, S.Wang and B.Wei, Multiplicative Zagreb and multiplicative hyper-Zagreb indices of Polycyclic Aromatic hydrocarbons, Benzenoid Systems, submitted.

15. M.Randić, On characterization of molecular branching, Journal of the American Chemical Society, 97(23) (1975) 6609-6615. 